IP Periodica Polytechnica

Transportation Engineering

45(4), pp. 181-185, 2017

https://doi.org/10.3311/PPtr. 10723

Creative Commons Attribution (i)

RESEARCH ARTICLE

\section{An Attitude Determination Method for Comprehensive Inspection Vehicle Based on Track Profile Registration}

\author{
Yumei Zhang ${ }^{1 *}$, Dalin Zhang ${ }^{1}$
}

Received 10 March 2017; accepted 18 March 2017

\begin{abstract}
The attitude of the comprehensive inspection vehicle is one of the important factors that affect the accuracy of the inspection of metro line infrastructure, meanwhile the metro environment restricts the employment of common attitude determination methods. A new method of attitude determination is presented in this paper, which takes the track as reference and employs non-contact measurement to acquire the track profile simulta-neously. By registration of measurement track profile and the standard track profile, the relative position between the vehicle and the track reference can be calculated; and the instantaneous attitude of the vehicle can be determined by the matrix inverse calculation. The performance of the method is verified by an experiment using the road-rail comprehensive inspection vehicle.
\end{abstract}

\section{Keywords}

comprehensive inspection vehicle, attitude determination, track profile, iterative closest point

\footnotetext{
${ }^{1}$ National Research Center of Railway Safety Assessment, Beijing Jiaotong University,

No.3 Shangyuancun, Haidian District, Beijing, P. R. China

"Corresponding author, e-mail: ymzh@bjtu.edu.cn
}

\section{Introduction}

As the rapid development of urban rail transportation, road-rail comprehensive inspection vehicle has been one of the important methods to inspection railway infrastructure parameters online, including track irregularity, tunnel profile, rail wear and so on. The inspection data captured by on-board device is usually observed and analysed in the coordinate system that built based on the horizontal plane determined by track top and the centreline of the track. However, due to the car vibration on multiple degrees of freedom caused by wheelrail dynamic forces, the on-board measurement equipment rigidly fixed on the inspection car will also be affected and the accuracy of inspection data will be greatly reduced (Yu et al., 2007). So studying the real time attitude determination method for the inspection vehicle and applied to dynamically compensate the attitude deviation of on-board inspection data, is significant to promotion the measurement accuracy of on board inspection systems.

Much research has been carried out on the study of motion object's attitude determination, strapdown inertial measurement, GPS based attitude determination, photogrammetry, multi-information fusion are the common way to determine the attitude of a motion platform (Noureldin et al., 2012). The widely used strapdown inertial measurement system (Onodera and Minura, 2012; Zhang and Chen, 2012) has high sample rate, short-term accuracy and reliability, and is independent from environment, but the error will accumulate as time which restricts the application on long term measurement situation. The GPS based attitude determination method (Han and Zhang, 2004; Xu et al., 2002) has long-term stability, no accumulation error, but has outrage problem in the underground or tunnel application. The photogrammetry for attitude determination (Kaiser et al., 2007; Caballero et al., 2006) is usually based on the daisy-chain thought, which doesn't rely on particular reference and determines attitude autonomously, has accumulation error as well and is susceptible to ambient light and vehicle speed.

As to the application of attitude determination in underground environment, Huang has proposed a method that employs track alignment data together with IMU for position and orientation 
determination, and conducted an experiment using a smallscale railway model (Hung et al., 2016). Hung's method relies on the known track alignment data which results in the loss of autonomy in attitude determination, and the imperfect of the track alignment data will lead to the reduce in accuracy.

Due to the characteristic of urban railway transportation, the existing common attitude determination method cannot simultaneously satisfy the accuracy, long-time measurement period, and the complex environment of the underground application. So this paper presents a new method based on track profile registration to determining the absolute attitude of the inspection car, which applies multiple 2D laser scanners to capture the dynamic track profile during the running of car, combined with the Iterative Closest Point (ICP) registration algorithm to calculate the real time attitude of the car.

In Section 2 we discuss the methods used for the attitude determination (2.1), give a brief introduction of the ICP algorithm used for track registration (2.2), as well as the calculation of car attitude according to the registration result (2.3). Section 3 describes the measurement system used to capture the track profile (3.1-3.3), the experiment process and result is presented and analysed (3.4). Then the paper ends with conclusions.

\section{Attitude determination method base on track profile registration}

\subsection{Basic principle of the attitude determination method}

The reference coordinate system of the on-board inspection equipment is built by the horizontal plane determined by track top and the centreline of the track, as shown in Fig. 1. The proposed method using track as reference, which is relatively static in the reference coordinate system. The on-board measurement equipment employs three laser scanners to simultaneously capture the track profile in real time. The original profile has three section allocated in separate's sensor coordinate systems, using calibration coefficient of three sensors' installation attitude in static state can transform the captured dynamic profile into the unified reference coordinate system.

When the car is stopping, the car attitude is same as that when calibrating, and the attitude of track profile in the reference coordinate system doesn't change. While during running of the comprehensive inspection car, the vibration resulting in the relative position change between measurement equipment and the track, which reflected by the attitude change of the measurement track profile in the unified reference coordinate system. And now take the standard track profile in the unified reference coordinate system as the target; employ the ICP algorithm to register the measurement track towards the target which produces transform matrix of the measurement track profile's attitude, and the car's attitude is just the inverse of the above transform.

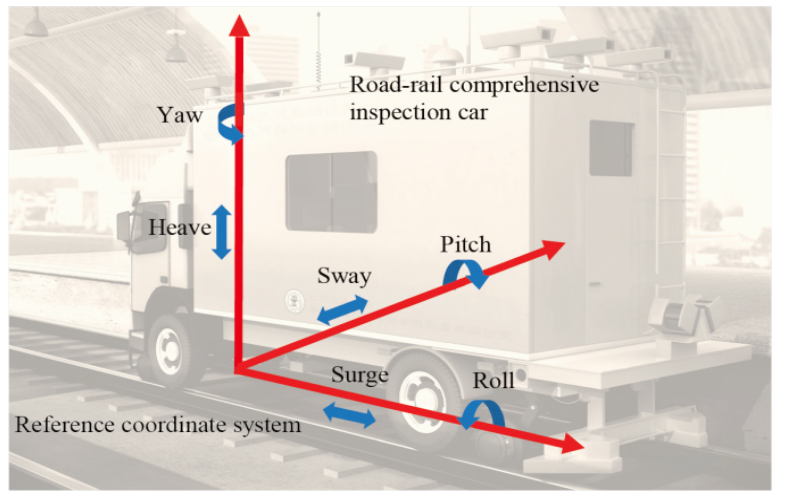

Fig. 1 Road-rail comprehensive inspection car and the reference coordinate system

\subsection{Iterative closest point algorithm}

Point set registration is the process of finding a spatial transformation that aligns two point sets observed from different perspectives of the same object. And ICP algorithm (Besl and McKay, 1992) is one of the most popular point set registration method, Given a source point set $\mathrm{P}=\left\{\vec{p}_{i}\right\}$ with $\mathrm{N}_{\mathrm{P}}$ points and target point set $\mathrm{X}=\left\{\vec{x}_{i}\right\}$ with $\mathrm{N}_{X}$ points, the ICP algorithm steps are:

Step 1. Initiate the iterative variable: the iterative number of times $\mathrm{k}=0$, the first intermediate point set $\mathrm{P}_{0}=\mathrm{P}$;

Step 2. Employ closest point set search algorithm (Mark et al., 2009) to search the closest point of each point in intermediate point set $\mathrm{P}_{k}$ from target point set $\mathrm{X}$ successively, and we get the closest point set $Y_{k}: \mathrm{Y}_{k}=C\left(P_{k}, X\right)$;

Step 3. Calculate the spatial transform matrix $\vec{q}_{k}$ that transforms the intermediate point set $P_{k}$ to the closest point set $Y_{k}$, as well as the residual $d_{k}: \vec{q}_{k}, d_{k}=Q\left(P_{0}, \mathrm{Y}_{k}\right)$;

Step 4. Using the transform matrix $\vec{q}_{k}$ to transform the intermediate point set $P_{k}$ and get new intermediate point set: $\mathrm{P}_{k+1}=\vec{q}_{k}\left(P_{0}\right)$, update the iterative number of times: $\mathrm{k}=\mathrm{k}+1$;

Step 5. If the residual changes significantly $\mathrm{d}_{k}-\mathrm{d}_{k+1}>\tau(\tau>0)$ then repeat the step 2-5, otherwise end the iterative process, and finally we get the rotation matrix $\mathrm{R}_{r}$ and the translation matrix $\mathrm{T}_{r}$ that transform the source point set to the target point set. The residual threshold $\tau$ determined by the accuracy request of the application.

\subsection{Attitude calculation according to the registration result}

After the registration of measurement track profile and the standard profile using ICP algorithm we got one 3 by 3 rotation matrix $\mathrm{R}_{r}$ and one 3 by 1 translation matrix $\mathrm{T}_{r}$, and the attitude matrix of the car are $\mathrm{R}_{V}=R_{r}^{\prime}, \mathrm{T}_{v}=-\mathrm{T}_{r}$. Then according to the spatial coordination transform Eq. (1), we can get the calculation formulas of the three rotation degree of freedom roll $\alpha$, yaw $\beta$ and pitch $\theta$ show as (2) - (4) 


$$
\begin{aligned}
& {\left[\begin{array}{c}
X \\
Y \\
Z
\end{array}\right]=\left[\begin{array}{c}
\cos \alpha \cos \beta \\
\sin \alpha \cos \beta \\
-\sin \beta
\end{array}\right.} \\
& \alpha=\tan ^{-1}\left(r_{21} / r_{11}\right) \\
& \beta=\tan ^{-1}\left(-r_{31} / \sqrt{r_{32}^{2}+r_{33}^{2}}\right) \\
& \gamma=\tan ^{-1}\left(r_{32} / r_{33}\right)
\end{aligned}
$$

Where $r_{i j}$ is the element in the ith row and jth column of the matrix $\mathrm{R}_{V}$.

\section{Experiment and results}

\subsection{The 2D laser scanner}

In this attitude determination method, the 2D laser scanner is employed to capture the track profile in real time. The principle of this sensor is shown as Fig. 2: the sensor emit a laser beam by the semiconductor laser generator, which becomes a light curtain after goes through the lens and form a band on the surface of the object; the camera lens collect the reflection light from the object which contains the profile information of the band and project it to the CMOS array; the signal processer analyse and deal with the cross-section image and output the profile point set of the object, which contains the $\mathrm{X}$ coordinate along the laser band direction and $\mathrm{Z}$ coordinate along the laser curtain direction.

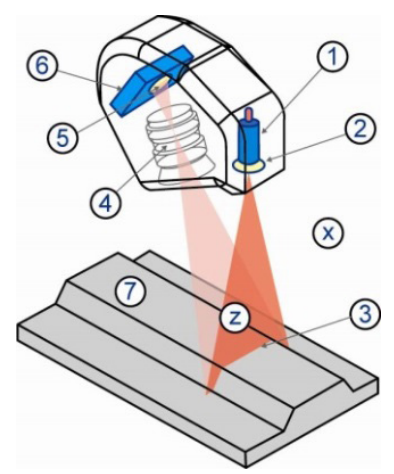

Fig. 2 Measurement principle of the 2D laser scanner

The laser scanner we chosen is the ZLDS200 laser scanner made by ZSY Group. The technical specifications are: the resolution along $\mathrm{X}$-axis is $128 / 256 / 512 / 1024$ points per profile; the sample rate is $100 \sim 1200 \mathrm{~Hz}$; as shown in Fig. 3, the measurement range is $65 \sim 170 \mathrm{~mm}$ along $\mathrm{X}$-axis with a linearity of $0.2 \%$, and $100 \sim 350 \mathrm{~mm}$ along $Z$-axis with a linearity of $0.15 \%$.

\subsection{The measurement system of track profile}

The measurement system of track profile takes the 2D laser scanner that based on the triangular as the measurement component. To get the complete profile of the rail track, three laser scanners is needed which positioned top left, directly above, top

$$
\left.\begin{array}{c}
\sin \alpha \sin \theta+\cos \alpha \sin \beta \cos \theta \\
-\cos \alpha \sin \theta+\sin \alpha \sin \beta \cos \theta \\
\cos \beta \cos \theta
\end{array}\right]\left[\begin{array}{l}
U \\
V \\
W
\end{array}\right]+\left[\begin{array}{c}
\Delta x \\
\Delta y \\
\Delta z
\end{array}\right]
$$

right separately. Scanners are configured working in the external synchronization trigger mode, with 1024 points per profile along $\mathrm{X}$-axis. The synchronization signal generator generates multiple synchronization pulse signals as the synchronous trigger signal of the scanners to make sure that the measurement track profiles are the same section.

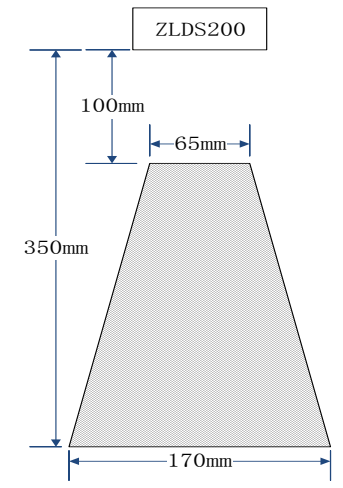

Fig. 3 Measurement range of ZLDS200

At the same time of track measuring, the Synchronous Information System of the comprehensive inspection car broadcast the timestamp and car position information through the RS485 bus, so that each inspection system has the unified time and position stamp which simplify the search and process of the inspect data. The overall structure of the track measurement system is as shown in Fig. 4.

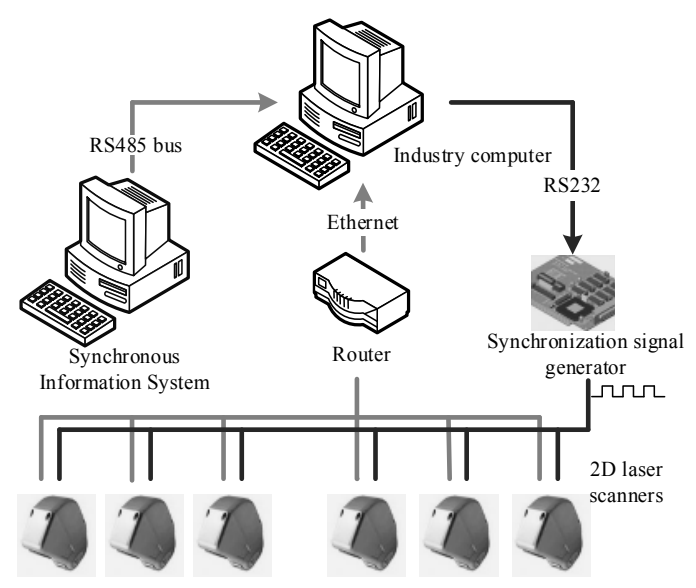

Fig. 4 Structure of the track measurement system

As shown in Fig. 5, the car attitude determination system is installed on the road-rail comprehensive inspection car, which is independently researched and developed by Beijing Jiaotong University, includes three 2D laser scanners, an interface box and one industrial compute. Three laser scanners' measurement data are pieced together to produce a complete profile of a single track. The laser scanners are mounted on the bracket 
that rigidly fixed on the car, the adjustment mechanism of the bracket allows adjustment of the attitude of laser scanners to make sure that the three laser band are in the same plane so that the three laser scanners are scanning the same section of the track. Fig. 6 presents the calibrated measurement track profile in the reference coordinate system.

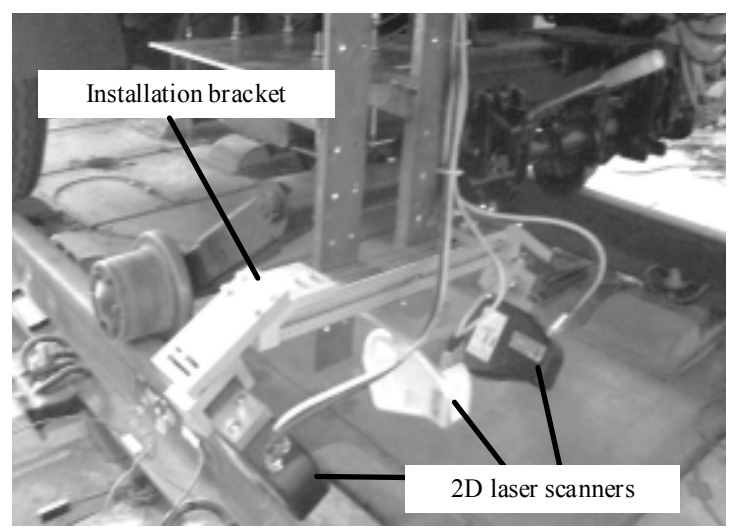

Fig. 5 Installation of the scanners

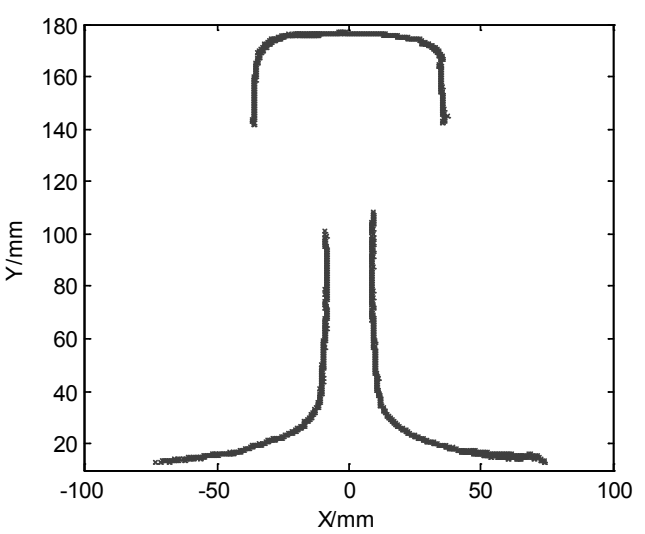

Fig. 6 Static measurement track profile

\subsection{Experiment process and result}

The static measurement data was used to verify the proposed attitude determination method, the verify process is as follows:

Step 1. The curve of the standard track profile is defined in the standard (GB2585-2007, 2007); take the point set of the discretized curve of standard track profile as the target point set X;

Step 2. Randomly generate groups of the five degrees of freedom of the car attitude (ignore the surge of the car which doesn't matter as to the dynamic compensation of the infrastructure inspection data), calculate the simulation transform matrix $\mathrm{R}_{s}$ and $\mathrm{T}_{S}$;

Referred to (Yu et al., 2007), the ranges of randomly generation of each attitude parameters are as follows: the scroll, yaw and pitch parameters are within the scope of $\pm 5^{\circ}$, the sway and heave parameters are within the scope of $\pm 50 \mathrm{~mm}$;

Step 3. Transforms the static track profile using $\mathrm{R}_{s}$ and $\mathrm{T}_{S}$ to get the measurement point set $\mathrm{P}$;

Step 4. Employs ICP algorithm to calculate the transformation matrix $R^{\prime}$ and $\mathrm{T}^{\prime}$, and calculate the attitude degree and translation of the car by using (2)-(4); compare the calculation parameters to generated ones.

Step 5. Repeat step 1 to step 4 to get more data to analyse the accuracy of the attitude determination method.

The verify process repeated eight times, and the error between calculation result and simulation parameters are shown as Table 1.

Table 1 Error of attitude determination

\begin{tabular}{lllllll}
\hline & $\varepsilon_{\alpha} / \mathrm{rad}$ & $\varepsilon_{\beta} / \mathrm{rad}$ & $\varepsilon_{\theta} / \mathrm{rad}$ & $\varepsilon_{X} / \mathrm{mm}$ & $\varepsilon_{Y} / \mathrm{mm}$ & $R M S / \mathrm{mm}$ \\
\hline 1 & 0.0055 & -0.0023 & -0.0001 & -0.27 & -1.38 & 0.5153 \\
2 & -0.0055 & 0.0005 & 0.0001 & 0.28 & 1.51 & 0.5153 \\
3 & -0.0026 & -0.0004 & -0.0007 & 0.26 & 1.17 & 0.5153 \\
4 & -0.0045 & 0.0015 & 0.0003 & 0.51 & 2.15 & 0.5153 \\
5 & -0.0005 & -0.0034 & -0.0038 & 0.52 & 1.37 & 0.5153 \\
6 & -0.0070 & -0.0052 & -0.0022 & -1.43 & -1.56 & 0.5153 \\
7 & -0.0043 & -0.0001 & 0.0003 & 0.46 & 1.01 & 0.5153 \\
8 & -0.0047 & -0.0003 & -0.0014 & 1.21 & -0.13 & 0.5157 \\
Avg. & -0.00295 & -0.0012 & -0.001 & 0.19 & 0.52 & 0.51535 \\
\hline stdev & 0.003923 & 0.002233 & 0.001459 & 0.772154 & 1.382593 & 0.000141 \\
\hline
\end{tabular}

As in the Table $1, \varepsilon_{\alpha}, \varepsilon_{\beta}, \varepsilon_{\theta}$ are the error of roll, yaw, pitch separately, $\varepsilon_{X}, \varepsilon_{Y}$ are the error of sway and heave, RMS is the root mean square of the point sets after registration. The first group of simulation track profiles that before and after registration are shown in Fig. 7.
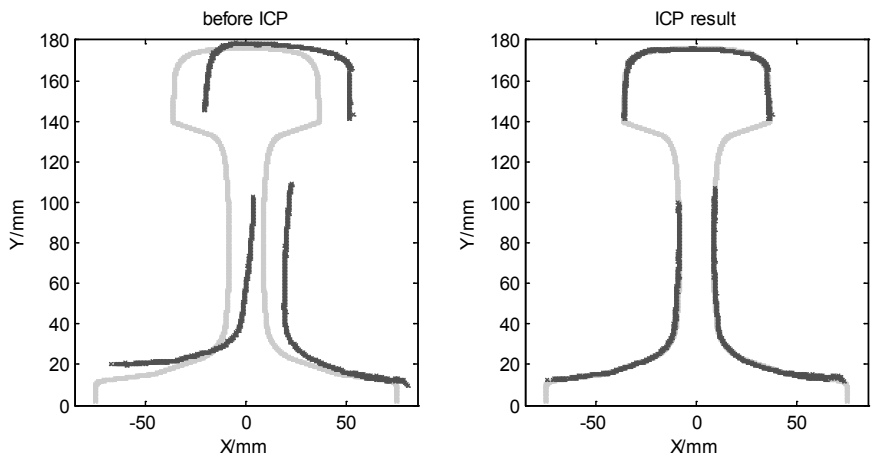

Fig. 7 Track profiles that before and after registration

\section{Conclusions}

This paper has suggested a new method to determinate the attitude of the comprehensive inspection car precisely and rapidly, for increasing the measurement accuracy of the on-board inspection system. The proposed method is aimed to applying to underground or tunnel environment that restricts the using of common attitude determination method. The detailed principle of the attitude determination method is presented in the paper. An experiment is conducted to verifying the performance of the method, the result shows that the method is applicable, the angular error is smaller than 0.02 degree $(1 \sigma)$ and the position error is smaller than $1.5 \mathrm{~mm}$. 
The new method produces the absolute attitude of the vehicle in the reference coordinate systems; has no accumulation errors; the laser based measurement is not susceptible to external environment; and can achieve attitude determination autonomously with high accuracy without rely on any data that related to particular line.

\section{Acknowledgement}

This work is sponsored by the National Natural Science Foundation of China under Grant No. 61502029. We are grateful to the anonymous reviewers for their comments on earlier drafts of this paper.

\section{References}

Besl, P. J., McKay, N. D. (1992). Method for registration of 3-D shapes. Proceedings of the SPIE. 1611, 586-606.

https://doi.org/10.1117/12.57955

de Berg, M., Cheong, O., van Kreveld, M., Overmars, M. (2008). Computational Geometry. Algorithms and Application. (3rd Edition). pp. 99-105. Springer, 2008.

https://doi.org/0.1007/978-3-540-77974-2

Caballero, F., Merino, L., Ferruz, J., Ollero, A. (2006). Improving vision-based planar motion estimation for unmanned aerial vehicles through online mosaicing. In: Proceedings of the 2006 IEEE International Conference on Robotics and Automation. ICRA 2006. Orlando, Florida, USA. May 15-19. 2006. pp. 2860-2865. https://doi.org/10.1109/ROBOT.2006.1642135

General Administration of Quality Supervision, Inspection and Quarantine of the PRC.(2007). GB2585-2007 Hot-rolled steel rails for railway.

Han X. D., Zhang J. (2004). GPS based attitude determination of vehicular platform. Geotechnical Investigation \& Surveying. (2), pp. 45-48.
Hung, R., King, B. A., Chen, W. (2016). A Method for the Positioning and Orientation of Rail-Bound Vehicles in Gnss-Free Environments. Isprs Annals of Photogrammetry, Remote Sensing and Spatial Information Sciences, III-1, pp. 135-142.

https://doi.org/10.5194/isprs-annals-III-1-135-2016

Kaiser, K., Gans, N., Dixon, W. (2007). Localization and control of an aerial vehicle through chained, vision-based pose reconstruction. In: $A C C$ ' 07 . American Control Conference, pp. 5934-5939. https://doi.org/10.1109/ACC.2007.4282896

Noureldin, A., Karamat, T. B., George, J. (2012). Fundamentals of inertial navigation, satellite-based positioning and their integreation. pp. 1-13. Springer Science \& Bussiness Media, 2012. https://doi.org/10.1007/978-3-642-30466-8

Onodera, R., Minura, N. (2012). Measurement of a vehicle motion using a new 6-DOF accelerometer. In: 2012 IEEE Sensors. Taipei, Taiwan. Oct. 2831, 2012. pp. 1068-1071. https://doi.org/10.1109/ICSENS.2012.6411095

Xu, J., Arslan, T., Wan, D., Wang, Q. (2002). GPS attitude determination using a genetic algorithm. In: Proceedings of the 2002 Congress on Evolutionary Computation, 2002. CEC ‘02., Honolulu, HI, 2002. pp. 998-1002. https://doi.org/10.1109/CEC.2002.1007061

Yu, Z. J., Zhu, L. Q., Zhao J. T. (2007). Study on system scheme for vehicular kinematical measurement of railway complete profile. Journal of Beijing Jiaotong University. 31(6), pp. 1-5. https://doi.org/10.3969/j.issn.1673-0291.2007.06.001

Yu Z. J., Zhao J. T., Zhu L. Q. (2007). Study on Measurement of Instantaneous Posture of Railway Vehicles. Journal of Electronic Measurement and Instrument. 21(5), pp. 45-48.

Zhang, Z. Y., Chen, J. Z. (2012). Research on running attitude detecting system for train bogie. China Measurement \& Test. 38(5), pp. 59-61. 